\title{
Laparoscopic Drainage of Retroperitoneal Hematoma
}

\author{
Anuradha R. Bhama, MD, Ronald J. Weigel, MD, PhD, Hui Sen Chong, MD \\ Department of Surgery, University of lowa, lowa City, IA, USA (all authors).
}

\begin{abstract}
Introduction: Operative intervention for retroperitoneal hematomas is rarely indicated, and treatment is usually conservative, including observation, transfusion, correction of coagulopathy, and coil embolization.

Case and Technique Description: A 75-year-old man underwent sigmoid resection and Hartmann's procedure for sigmoid volvulus. Ten days postoperatively, he presented with a large left-sided retroperitoneal hematoma and a supratherapeutic international normalized ratio. After resuscitation and reversal of coagulopathy, he continued to bleed and required vasopressor support. He was not a candidate for procedures requiring intravenous contrast given new-onset acute renal failure. He was taken to the operating room for laparoscopic drainage of the hematoma with a total retroperitoneal approach. Several small bleeding points were identified and cauterized. The patient's hemodynamic status and hemoglobin stabilized postoperatively. He did not experience any further bleeding complications. His renal impairment resolved.
\end{abstract}

Conclusion: Unstable patients with retroperitoneal hematoma, who are not candidates for coil embolization, can be treated successfully with laparoscopic drainage with a retroperitoneal approach.

Key Words: Postoperative hemorrhage, Laparoscopy, Retroperitoneal hematoma

\footnotetext{
Citation Bhama AR, Weigel RJ, Chong HS. Laparoscopic drainage of retroperitoneal hematoma. CRSLS e2014.00054. DOI: 10.4293/CRSLS.2014.00054.

Copyright (c) 2015 by SLS, Society of Laparoendoscopic Surgeons. This is an open-access article distributed under the terms of the Creative Commons Attribution-Noncommercial-ShareAlike 3.0 Unported license, which permits unrestricted noncommercial use, distribution, and reproduction in any medium, provided the original author and source are credited.

Address correspondence to: Hui Sen Chong, MD, University of Iowa, Department of Surgery, 200 Hawkins Drive, 4630 JCP, Iowa City, IA 52242, USA, Tel: 319-356-7675, Fax: 319-356-4609, E-mail: huisen-chong@uiowa.edu.
}

\section{INTRODUCTION}

Retroperitoneal hematoma is a rare but potentially lifethreatening condition. Often it can be treated with conservative medical management without requiring operative intervention. This includes fluid and blood resuscitation, correction of coagulopathy, and intensive care monitoring. There are rare situations in which medical management is not successful and the patient continues to hemorrhage. In these instances, the bleeding can often be diagnosed angiographically and treated with embolization. In rare instances, surgical intervention is necessary; this entails open exploration of the retroperitoneum, evacuation of hematoma, achievement of hemostasis, and drainage of the retroperitoneum. The evacuation of a retroperitoneal hematoma using a laparoscopic technique has never been described.

\section{CASE DESCRIPTION}

The patient was a 75-year-old male who initially presented to our institution with hemorrhagic shock and peritonitis. His medical history was significant for atrial fibrillation and chronic anticoagulation. His international normalized ratio on presentation was 4.7 , which was reversed with fresh frozen plasma and vitamin K. He was taken to the operating room emergently for exploration. Intraoperative findings were significant for sigmoid volvulus with associated mesenteric hemorrhage. The patient underwent a Hartmann's procedure, and the remainder of his hospital course was uneventful. He was restarted on warfarin and was discharged home on postoperative day 7 .

Four days after discharge, the patient presented again with hemorrhagic shock. He was found to have a large retroperitoneal hematoma with an international normal- 


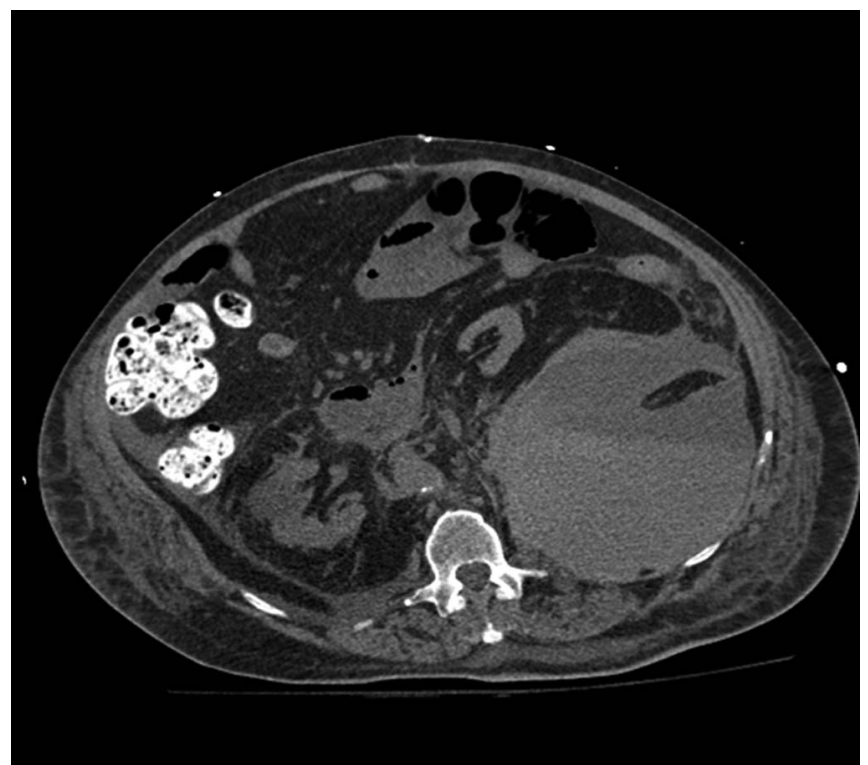

Figure 1. Layering retroperitoneal hematoma suggestive of ongoing bleeding.

ized ratio of 4.2 and hemoglobin of $5.8 \mathrm{~g} / \mathrm{dL}$. His coagulopathy was reversed with fresh frozen plasma and vitamin $\mathrm{K}$, and he was resuscitated with fluids and packed red blood cell transfusion. On admission, he was also found to have acute renal insufficiency, with a creatinine level of $2.8 \mathrm{mg} / \mathrm{dL}$. The patient's retroperitoneal hematoma was managed with correction of coagulopathy, transfusion of blood, and fluid resuscitation. Within 24 hours of admission, the patient continued to have downward-trending hemoglobin, and a repeat noncontrast computed tomographic scan demonstrated enlargement and further layering of the retroperitoneal hematoma, suggestive of an ongoing bleed (Figure 1). Additionally, the patient developed weakness of the left lower extremity hip flexor muscles, concerning for femoral neuropathy due to compression from the hematoma. Given the patient's acute renal failure, angiographic intervention with contrast administration was not an option, and the patient was taken to the operating room for hematoma evacuation, which was performed laparoscopically.

The patient was positioned in the right lateral decubitus position, as demonstrated in Figure 2. The prior computed tomographic images were used to identify a point of entry just under the 12th rib in the midaxillary line (Figure 3). The first port was placed in this location using the optical trocar method to enter the retroperitoneal space. On entrance to the retroperitoneum, there was

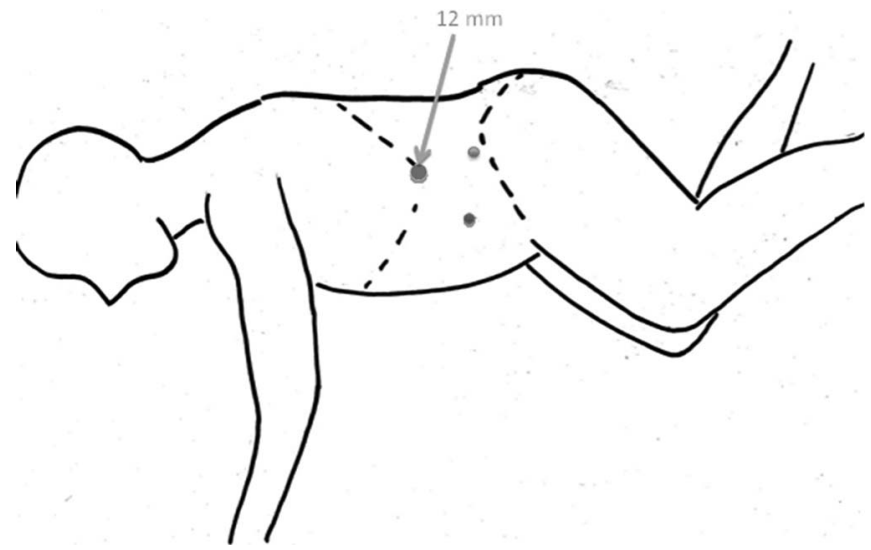

Figure 2. Patient positioning in operating room and port placement.

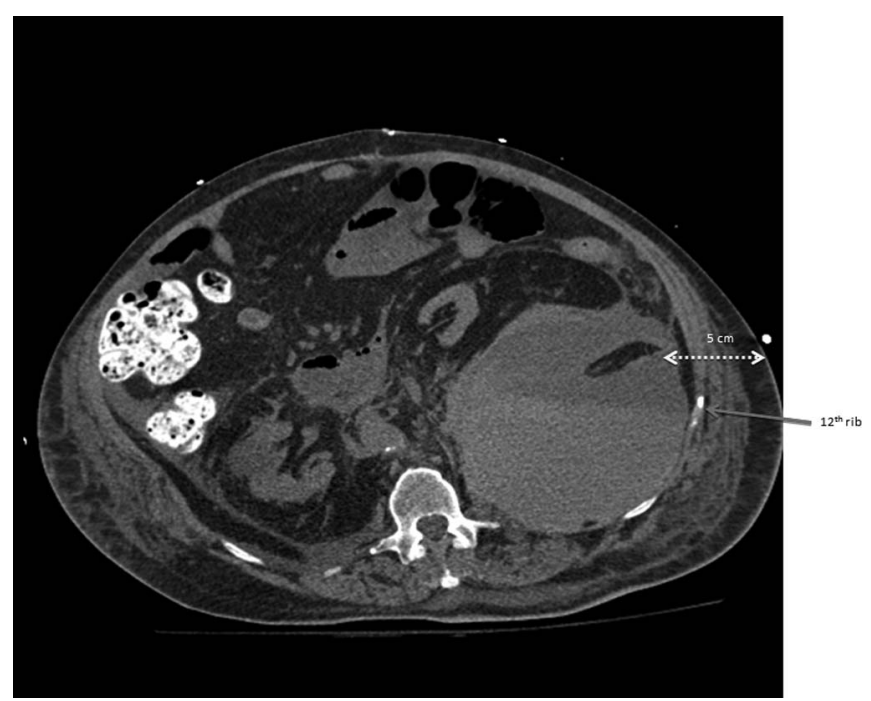

Figure 3. Identification of initial port placement location just under the 12th rib.

immediate evacuation of approximately $700 \mathrm{~mL}$ of blood through the trocar. The evacuated space was then insufflated with carbon dioxide to a pressure of $15 \mathrm{~mm} \mathrm{Hg}$ and explored with the laparoscope. The retroperitoneal cavity was examined, and additional ports were placed: a 5-mm port in the midaxillary line just above the anterior superior iliac spine, and another $5-\mathrm{mm}$ port in the anterior axillary line.

The remaining blood clot was evacuated with the suction irrigator device. There were 3 areas of active oozing, with one originating from a lumbar vessel. All bleeders were controlled with electrocautery, and $15 \mathrm{~mL}$ of fibrin sealant was infiltrated into the retroperitoneal area. Two 19-Fr 
round-channel drains were left within the retroperitoneal cavity.

Postoperatively, the patient's hemoglobin stabilized and his renal function normalized. He no longer required further blood transfusion, and the drains were removed on postoperative day 3 . The patient was discharged to a skilled nursing facility on postoperative day 7 . Given that the patient experienced 2 life-threatening bleeding events, the decision was made to discontinue anticoagulation therapy indefinitely.

\section{DISCUSSION}

This report is a case of laparoscopic management of a retroperitoneal hematoma. Retroperitoneal hematoma is a rare but potentially life-threatening condition that can occur in up to $0.6 \%$ of patients on oral anticoagulation therapy. ${ }^{1}$ There is no consensus among clinicians as to the optimal management strategy for treatment of this condition. Most cases can be managed conservatively. Stable patients are most always treated conservatively with close monitoring, blood transfusion, and reversal of coagulopathy. ${ }^{2,3}$ Patients with hemodynamic instability and/or falling hematocrit levels require intervention to cease the hemorrhage. Most often, this can be done by using angiographic techniques with embolization or open operative intervention. 4,5

Indications for open evacuation of retroperitoneal hematoma include persistent hemodynamic instability despite adequate resuscitation, abdominal compartment syndrome, or neuropathy due to compression of the femoral nerve. To evacuate the retroperitoneal hematoma, 1 of 2 open approaches may be chosen. The first approach is to make an ipsilateral hockey-stick incision to gain access to the retroperitoneal cavity. The second approach involves a midline laparotomy. In the case of patients with concurrent abdominal compartment syndrome, this would allow evacuation of retroperitoneal hematoma along with alleviation of elevated intra-abdominal pressure. The abdomen can be closed with a temporary dressing, and the patient can be brought back to the operating room at a later date for reexploration with fascia closure.

In our patient, the new onset of acute kidney injury precluded the use of intravenous contrast, leaving open or laparoscopic surgical intervention as the remaining treatment options. Our patient required intervention not only to cease the ongoing hemorrhage but also to relieve compressive symptoms from the hematoma.
Femoral neuropathy from retroperitoneal hematoma has been described in the literature, and surgical decompression has been shown to hasten recovery and minimize residual neurologic deficits. ${ }^{6,7}$ Additionally, compression of the renal vein and inferior vena cava may have contributed to low cardiac output and hence exacerbation of acute renal failure. The decision to proceed with laparoscopic drainage was based on the desire to avoid the morbidity of an open incision in this critically ill patient while still successfully addressing the life-threatening hemorrhage.

Strategic port placement was critical in successfully completing the procedure. Using preoperative imaging, we were able to determine a safe entry point for the first port directly into the hematoma. This avoided injury to vital retroperitoneal structures, which were displaced by the hematoma. Upon placement, the initial trocar was used as a sump for drainage of any nonclotted blood. Following partial decompression of the hematoma, we were able to insufflate the retroperitoneum to allow visualization and additional port placement. Three minor bleeding points were successfully identified because of the magnified view offered by laparoscopy. On completion of the case, 2 of the port sites served as exit points for the surgical drains.

Risks of a laparoscopic approach must be considered. One could hypothesize that releasing the tamponade from the hematoma could result in life-threatening hemorrhage. However, most cases of spontaneous retroperitoneal hematomas from supratherapeutic anticoagulation are a result of diffuse minor oozing rather than pulsatile bleeding from a major vessel.

Approaching this case from a laparoscopic standpoint allowed a safe, relatively fast, and effective treatment of retroperitoneal hematoma that was not responding to medical management. A direct retroperitoneal laparoscopic approach allows the surgical team to avoid intra-abdominal adhesions, with the added benefit of avoiding a large incision associated with an open procedure.

\section{CONCLUSION}

Angiographic embolization of bleeders and open evacuation of retroperitoneal hematoma have been well documented in the past. We have described the first laparoscopic drainage of retroperitoneal hematoma in a 
patient. This technique, though novel, was successful at managing this condition. Further studies are required to study this technique in comparison with the open technique.

\section{References:}

1. Estivill Palleja X, Domingo P, Fontcuberta J, Felez J. Spontaneous retroperitoneal hemorrhage during oral anticoagulant therapy. Arch Intern Med. 1985;145(8):1531-1534.

2. Kent KC, Moscucci M, Mansour KA, et al. Retroperitoneal hematoma after cardiac catheterization: prevalence, risk factors, and optimal management. J Vasc Surg. 1994;20(6):905910 .

3. Chan YC, Morales JP, Reidy JF, Taylor PR. Management of spontaneous and iatrogenic retroperitoneal haemorrhage: con- servative management, endovascular intervention or open surgery? Int J Clin Pract. 2008;62(10):1604-1613.

4. Akpinar E, Peynircioglu B, Turkbey B, Cil BE, Balkanci F. Endovascular management of life-threatening retroperitoneal bleeding. ANZ J Surg. 2008;78(8):683-687.

5. Isokangas JM, Perala JM. Endovascular embolization of spontaneous retroperitoneal hemorrhage secondary to anticoagulant treatment. Cardiovasc Intervent Radiol. 2004;27(6):607-611.

6. Parmer SS, Carpenter JP, Fairman RM, Velazquez OC, Mitchell ME. Femoral neuropathy following retroperitoneal hemorrhage: case series and review of the literature. Ann Vasc Surg. 2006;20(4):536-540.

7. Zarranz JJ, Salisachs P. Femoral neuropathy due to compression by retroperitoneal haemorrhage: a modern evaluation. J Neurol Sci. 1979;43(3):479-482. 